\title{
Retours contraints de migrants internationaux au Sénégal : dilemmes familiaux face à la maladie mentale
}

Forced Returns of International Migrants in Senegal: Family Dilemmas Facing Mental Illness

Retornos forzosos de migrantes internacionales a Senegal: dilemas familiares frente a la enfermedad mental

\section{Véronique Petit}

\section{(2) OpenEdition}

\section{Journals}

Édition électronique

URL : https://journals.openedition.org/remi/10901

DOI : 10.4000/remi.10901

ISSN : $1777-5418$

Traduction(s) :

Forced Returns of International Migrants in Senegal: Family Dilemmas Facing Mental Illness - URL : https://journals.openedition.org/remi/13127 [en]

Éditeur

Université de Poitiers

Édition imprimée

Date de publication : 1 octobre 2018

Pagination : 131-158

ISBN : 979-10-90426-62-7

ISSN : 0765-0752

Référence électronique

Véronique Petit, «Retours contraints de migrants internationaux au Sénégal : dilemmes familiaux face à la maladie mentale », Revue européenne des migrations internationales [En ligne], vol. 34 - $n^{\circ} 2$ et 3 । 2018, mis en ligne le 01 janvier 2021, consulté le 14 avril 2022. URL : http://journals.openedition.org/ remi/10901 ; DOI : https://doi.org/10.4000/remi.10901 


\section{Retours contraints de migrants internationaux au Sénégal : dilemmes familiaux face à la maladie mentale}

\section{Véronique Petit ${ }^{1}$}

Cet article rend compte de la situation de certains migrants de retour dans leur pays d'origine, le Sénégal, du fait d'une pathologie mentale alors que ce voyage n'est ni anticipé ni souhaité par les acteurs impliqués. Cette situation spécifique offre un éclairage sur une forme de retours contraints indépendamment des rapatriements, des échecs dans la réalisation de la migration ou d'un retour programmé. Des données originales permettent d'interroger l'impact de la santé mentale sur les parcours migratoires et ce qu'il révèle de l'investissement de la solidarité familiale dans la réalisation du projet migratoire. Les observations et les récits questionnent la force du statut de migrant dans la société sénégalaise et sa possible érosion alors qu'il est présenté comme une "very very VIP" et qu'il incarne une des figures majeures de la réussite. Les migrants affectés par une maladie mentale peuvent revenir par leurs propres moyens ou être rapatriés pour des raisons sanitaires afin d'être soignés au Sénégal lorsque survient une dégradation de leur état psychique. La pathologie peut préexister à la vie en migration, mais cette dernière peut constituer un contexte favorable à son émergence ou à l'aggravation de l'état de santé du fait des conditions quotidiennes d'existence du migrant.

Notre regard se concentre sur les premiers temps du retour au Sénégal et les questions que pose la prise en charge thérapeutique du migrant aux familles confrontées au dilemme suivant : comment concilier le devoir moral de soigner le migrant qui contribue aux ressources de la famille tout en tentant de limiter la durée de la cure afin de pousser le migrant à reprendre au plus vite le chemin de la migration? Les décisions des familles sont à replacer dans un contexte social et thérapeutique spécifique : la stigmatisation de la folie dans la société sénégalaise, le coût financier des soins ainsi que la durée du traitement. La confrontation entre l'état de santé du migrant et les attentes multiples qui pèsent sur lui pose aussi la question de la reconnaissance du statut de malade et la

\footnotetext{
1 Démographe, Professeur, CEPED, Université Paris Descartes/IRD, 45 rue des SaintsPères 75006 Paris; veronique.petit@parisdescartes.fr

L'auteure remercie l'ensemble des services psychiatriques publics et privés au Sénégal, leurs personnels, les institutions et associations, où elle a pu conduire cette enquête, pour leur accueil et leur patience. Cette recherche ne pourrait avoir lieu sans la confiance des personnes malades et celle de leur entourage familial et amical, qu'ils trouvent ici l'expression de notre gratitude.
} 
légitimité de la plainte alors que le migrant doit sans cesse renouveler la preuve de sa performativité en tant que corps au travail. Les données proviennent d'une enquête en cours plus vaste consacrée à l'accès des individus aux soins en santé mentale au Sénégal, les migrants internationaux ne constituant qu'un groupe au sein de la population générale.

Des travaux plus anciens ont déjà évoqué les conséquences négatives de la migration sur la santé mentale et les dysfonctionnements intra familiaux qui étaient apparus dès les années 1960 dans les pays d'émigration situés sur le pourtour du Bassin méditerranéen (Charbit et Bertrand, 1985). Dans le cas du Sénégal, on mentionnera l'étude de Collomb et Ayats (1962 : 595-596) qui évoquent "une pathologie du retour" en se référant aux situations des migrants "qui sans atteindre au bouleversement psychotique, ont des difficultés à réintégrer leur groupe d'origine. Elles sont liées davantage à un sentiment de faute, conséquence de la non-observation des règles du groupe, qu'à une désorganisation profonde". Nous n'évoquons pas ici la fréquentation par les enfants et les épouses de migrants des services psychiatriques alors que des observations démontrent qu'ils sont eux aussi affectés par les conditions de vie (absence, attente, secrets, dépendance) qu'impose l'expérience migratoire à ceux qui restent et sont exclus de la circulation. Le retour ou plus exactement un retour sans cesse différé pour des raisons personnelles ou en raison des effets des politiques migratoires produit des effets morbides sur la famille du migrant restée au pays. Ces situations restent à étudier en Afrique subsaharienne indépendamment des cas des populations réfugiées ou des rapatriements forcés de jeunes subsahariens ayant échoué à atteindre I'Union européenne. Ces configurations familiales complexes méritent d'être l'objet d'un traitement approfondi, mais elles sont à l'évidence complémentaires de celles qui vont être décrites dans cet article.

Rappelons également brièvement l'importance des migrations et des mobilités dans la société sénégalaise. Historiquement, le Sénégal a été avant la période coloniale un des centres du commerce reliant l'Europe, I'Afrique et l'Amérique compte tenu de sa situation géographique (façade atlantique aux marges des royaumes africains). À partir de 1895, la colonisation et l'inclusion dans l'Afrique-Occidentale française (AOF) entraînent la mobilité de différentes catégories : des hommes recrutés dans les régiments des tirailleurs sénégalais, grâce à la scolarisation des fonctionnaires au sein de l'AOF, des commerçants, des marins. Après l'indépendance en 1960, l'émigration prend une nouvelle ampleur et les flux sont orientés vers la France et des pays africains (Ghana, Côte d'Ivoire, Gabon, République Démocratique du Congo) dans un contexte économique et politique favorable. La demande de main-d'œuvre extérieure se nourrit de la crise que vit le monde rural et de la reconversion qu'opère parallèlement la confrérie des Mourides (Diop, 1980 et 1981). Celle-ci, grâce au commerce international et aux investissements qu'elle réalise en s'appuyant sur la diaspora et ses réseaux, devient une actrice centrale des migrations. Le choc pétrolier et ses conséquences économiques et politiques, la fermeture de certaines destinations africaines conduit à partir de la fin des années 1970 à une réorientation et une diversification des flux migratoires vers I'Italie, l'Espagne, les États-Unis, le Canada, les Émirats du golfe, certains migrants restent bloqués dans les pays du Maghreb faute de parvenir à atteindre I'Union européenne. Les profils des migrants se sont diversifiés, impliquant davantage de femmes, d'étudiants et 
de diplômés. En raison des investissements et des transferts, l'émigration joue un rôle socioéconomique de premier plan. Elle n'est pas non plus complètement définitive, puisque selon les estimations la moitié des émigrés sénégalais reviennent dans leur pays après dix années de vie à l'étranger (Lessault et al., 2015).

\section{Théoriser les relations entre le migrant et sa famille au fil du parcours migratoire}

Si la prise de décision d'émigrer a été largement théorisée, la question du retour l'a été moindrement ou elle reste latente dans les théories. Sans doute parce qu'initialement les théoriciens adoptaient implicitement le point de vue des pays d'immigration du Nord (Amérique, Europe), et qu'il leur importait de modéliser les motivations des migrants afin de comprendre quelles pouvaient être leurs contributions aux économies et leurs capacités "d'intégration" notamment grâce à l'activité professionnelle aux sociétés qu'ils rejoignaient à cette époque de croissance. Cette prise de décision se résume à un calcul, à une comparaison mettant en perspective les avantages et les coûts à rester ou à partir (Lee, 1966; Harris et Todaro, 1970). Dans cette perspective, le migrant - acteur individuel - est un être rationnel, un homo oeconomicus qui réagit aux déterminants structurels (différentiels de niveaux de salaires, opportunités d'emplois) qui orientent les flux migratoires dans une perspective de mobilité sociale. Le migrant maximise son intérêt en choisissant une destination qui lui permet de satisfaire ses attentes et de valoriser son capital humain (Becker, 1964).

À partir des années 1990, la nouvelle économie des migrations en se fondant sur la micro-économie des familles (Becker, 1964) renouvèle ce cadre théorique. Les migrations internationales sont analysées non plus comme une stratégie de maximisation des gains, mais comme une stratégie de diversification des risques afin de les minimiser. Les économies depuis le milieu des années 1970 sont entrées dans une période de crise et d'instabilité. Les acteurs doivent donc prendre leurs décisions dans un contexte économique, sociopolitique et environnemental caractérisé par un fort degré d'incertitude. Les coûts et les bénéfices doivent être calculés, de même que les probabilités d'échec ou de succès, pour l'ensemble du ménage et non plus seulement pour le seul individu migrant. La migration elle-même est une stratégie de gestion du risque (Stark, 1984). Autre avancée théorique, le processus de décision d'émigrer et donc la nature du projet migratoire ne sont plus considérés comme une initiative purement individuelle, mais davantage comme un projet élaboré et décidé au niveau de la famille. La famille est qualifiée par Faist (1997) de «the crucial meso level» et devient déterminante dans les recherches sur les migrations internationales à travers l'analyse des réseaux et des diasporas. La structure familiale, les rôles sexuels dans l'organisation du travail et les relations de parenté élargissent ce contexte décisionnel. Dès lors, la famille joue le rôle d'une institution qui effectue le choix d'un acteur migrant et qui ensuite soutient la réalisation du projet migratoire en mobilisant ses multiples ressources que ce soit dans les pays de départ ou d'arrivée. En adoptant le point de vue interne des familles, on comprend pourquoi la migration de certains de ses membres est davantage souhaitée que d'autres. 
La solidarité (redistribution et réciprocité) et les liens qui unissent le migrant à sa famille sont pensés dans le cadre d'une économie morale définie "comme un système d'échanges de biens et de prestations, articulé " à "un système de normes et d'obligations qui orientent les jugements et les actes, distingue ce qui se fait et ne se fait pas" au sein d'une collectivité d'appartenance (Fassin, 2009 : 1243). Le migrant est redevable à sa famille qui s'est mobilisée pour permettre la réalisation du projet migratoire à travers la logique de redistribution qui s'est mise en place autour de lui. À travers les différentes formes que peut prendre la rente migratoire, le migrant contribue à la santé, à l'éducation, aux activités économiques des membres de sa parenté. Cette rente, même si le terme peut paraître abusif par la régularité et la dépendance qu'il engage, implique que le retour doit être anticipé (investissements immobiliers, fonciers, activité professionnelle, création d'entreprises, etc.). De retour, le migrant est supposé capitaliser les ressources matérielles et immatérielles qu'il a acquises et accumulées durant son expérience migratoire. Dans cette perspective, son retour est attendu, légitimé, fêté, car il s'ancre dans une dynamique de réussite et de promotion sociale. Néanmoins si cette vision du retour comme une étape logique du parcours migratoire correspond aux profils de certains migrants (entrepreneurs, étudiants ayant acquis une formation, retraités), d'autres échappent à ce parcours idéal ou idéalisé pour diverses raisons.

Dans cette perspective, Guilmoto et Sandron (2001) définissent la notion de contrat migratoire entre la famille et son migrant, la première investit sur le second, qui doit rendre de manière différée les investissements dont il a été I'objet. Ce contrat est atemporel, son caractère implicite et tacite rend possible une renégociation entre les parties prenantes au fur et à mesure que la vie en migration se déroule en fonction des contraintes et des opportunités dans les régions de départ et d'arrivée. Les transferts effectués garantissent au migrant un "droit au retour" en lui permettant de conserver un accès au foncier, au marché matrimonial et à la vie politique (Stark, 1984; Stark et Lucas, 1988). Dans une perspective anthropologique, Dia (2009) forge la notion d'«esthétique du mensonge " afin de décrire le jeu en miroir des acteurs familiaux ici et là-bas qui travestissent la réalité de leurs existences, du côté des migrants afin de résister aux multiples sollicitations dont ils sont constamment l'objet et du côté de leur parenté afin de tirer le meilleur avantage de la rente migratoire.

Dans le contexte de crise économique précédemment évoqué, les solidarités deviennent de plus en plus sélectives et choisies dans les pays en développement (Calvès et Marcoux, 2007; Marie, 2007; Marie et al., 1997). Parallèlement du fait de la transition démographique et des changements socioéconomiques les structures familiales se transforment, la famille élargie se délitant au profit d'une nucléarisation des familles et favorisant les processus d'individualisation. L'intégration du niveau méso conduit également à expliquer l'accroissement de la migration au sein de sociétés, celle-ci devenant un modèle de réussite, et sa reproduction à travers l'émergence d'une culture de la migration. Le désir de migration et les imaginaires migratoires sont alimentés par les récits et les pratiques des migrants lors de leurs retours (Massey et al., 1987). Loin de freiner les migrations internationales, le développement contribue aussi à l'extension et à la diversification des formes de mobilités (De Haas et Fokkema, 2011; De Haas, 2005; Skeldon, 2008), car davantage de personnes disposent du capital nécessaire à l'émigration. Ce cadre théorique est pensé dans le contexte d'une 
migration "réussie" et régulière, dans un contexte où le migrant s'intègre socialement grâce au marché de l'emploi. Il est ainsi en mesure d'effectuer des remises qui lui permettent à la fois de rembourser les investissements dont il a bénéficié afin d'émigrer, d'entretenir des liens avec sa famille et de préparer un éventuel retour dans son pays d'origine. États et institutions promeuvent le migrant comme acteur du développement à travers leurs politiques.

Au cours d'une enquête de démographie anthropologique (Petit, 2013; Petit et al., 2019) sur l'accès et l'adhésion aux soins en santé mentale au Sénégal dans des unités de soins psychiatriques, nous avons croisé soit directement la situation de certains migrants de retour hospitalisés, soit indirectement à travers des récits faisant état de situations comparables à celles que nous observions. Leurs histoires émanaient des différentes catégories de personnel soignant, du personnel administratif, de patients eux-mêmes et de leurs parents. La fréquence de ces situations n'est pas quantifiable faute de données statistiques et en raison du contexte social qui pousse à leur dissimulation.

Les données présentées ici sont relatives à quinze migrants sénégalais interrogés dans plusieurs unités de soins. Ils sont très majoritairement en situation régulière avec une activité salariée en France, en Italie, au Canada, aux ÉtatsUnis ou en Belgique. Trois d'entre eux sont étudiants et occupent un emploi parallèlement à leur formation. Le plus fréquemment ils sont célibataires ou seuls ayant laissé leurs épouses au Sénégal. Ils travaillent dans la restauration, les services (gardiennage et sécurité, techniciens de surface), et le bâtiment. Affectés par une pathologie mentale, ils préfèrent fréquemment dans un premier temps recourir à des traitements traditionnels auprès de marabouts immigrés (Hamès, 2003) ou par l'intermédiaire de leurs réseaux ils se font envoyer médicaments et gris-gris élaborés par des tradipraticiens. Certains se décident seuls à consulter en psychiatrie, d'autres sont orientés par leur médecin généraliste ou sont poussés par une relation influente à effectuer cette démarche. Dans d'autres cas, ils sont hospitalisés en situation de crise.

Leur état pathologique se répercute sur leur situation économique et sociale qui se dégrade faute d'un suivi psychiatrique régulier et d'un soutien familial suffisant. Au Sénégal comme en situation migratoire, I'assignation au statut de "fou" ou de "malade mental" génère une forme de distanciation sociale. Finalement, ils font l'objet d'un rapatriement sanitaire soit à leur demande, soit à la demande de leur famille qui s'inquiète, en particulier lorsqu'une hospitalisation se prolonge, qu'aucun parent $n^{\prime}$ assiste le malade de manière prolongée, et que la maladie engendre une perte de revenus. II n'est pas toujours aisé dans les discussions d'identifier clairement qui prend cette décision, car elle relève davantage d'un processus d'échanges que d'un acte ponctuel. D'autres malades ayant commencé un traitement psychiatrique souhaitent rentrer au Sénégal afin de poursuivre leur cure auprès d'un psychiatre sénégalais. Ils considèrent en effet qu'un médecin sénégalais aura une meilleure compréhension de leurs problèmes de santé en raison d'une proximité socioculturelle qu'ils ne partagent pas avec les psychiatres des pays d'arrivée du fait des représentations et des interprétations de la maladie (Storper-Perez, 1976; Zempléni, 1985; Ortigues 
et Ortigues, 1966)2. Parfois, ce sont les psychiatres qui les suivent en Europe, notamment des médecins originaires du Maghreb, qui les encouragent à rentrer chez eux, arguant qu'ils y seront mieux entourés par leur famille et que la chaleur et la solidarité familiale contribueront à leur rétablissement. Cette vision idéalisant simultanément la prise en charge thérapeutique et la solidarité familiale au Sénégal est contestée par l'ensemble des acteurs. Pour les migrants malades, "les médecins au Nord ne connaissent pas les réalités des familles ici [c'est-à-dire au Sénégal], la maladie mentale ça t'isole, te coupe, même nous qui sommes d'une famille d'éduqués, avec des enseignants, des intellectuels" (Femme, universitaire, 46 ans). Cependant, certains migrants expriment clairement la volonté de rentrer au pays afin d'être pris en charge par leur famille durant cet épisode morbide, face aux difficultés qu'ils rencontrent pour assumer et conjuguer leurs obligations professionnelles et thérapeutiques dans un contexte où leur environnement social s'effrite face aux représentations liées à la folie. Dans l'ensemble de ces cas, le retour au pays n'avait pas été anticipé puisqu'il ne représentait pas une intention à courte échéance. La situation psychique et ses multiples conséquences socioéconomiques l'imposent. La gestion de la maladie et les choix thérapeutiques engageant l'avenir du migrant constituent dès lors un puissant révélateur des dynamiques familiales.

\section{Contexte psychiatrique au Sénégal et dispositif d'enquête}

\section{La psychiatrie culturelle}

Le Sénégal dispose d'une offre en santé mentale héritée de la colonisation française ${ }^{3}$. La France a été le premier État au monde à établir une législation, la loi du 30 juin 1838, afin de protéger la société et de garantir des droits aux personnes malades. Les effets de cette loi ne seront pas identiques en métropole et dans l'empire colonial, de surcroît des différences émergent entre les colonies au fil du temps. Au début du XXe siècle, les médecins coloniaux constatent que le système de santé dans l'empire colonial français est en retard comparé aux possessions anglaises et hollandaises. Avant 1897, les personnes désignées comme folles dans les colonies françaises font l'objet d'une procédure judiciaire, car l'autorité administrative dispose du pouvoir d'interner toute personne perturbant l'ordre public. L'avis médical demandé dans le cas des personnes présentées comme folles n'implique aucune des considérations sociales et thérapeutiques liées à la loi de 1838 appliquée en métropole. Cette pratique judiciaire d'enfermement montre ses limites, et à partir de 1896 I'administration coloniale choisit de manière pragmatique d'exiler les fous vers des asiles en métropole. En 1912, le Rapport sur les aliénés aux colonies rédigé par les docteurs Reboul et Régis dénonce la situation sanitaire dans les colonies françaises. Ils défendent avec force l'idée selon laquelle la France a le devoir moral d'offrir des soins aux personnes aliénées dans les colonies. Cette prise

\footnotetext{
2 Ce n'est pas l'objet de ce travail, mais les entretiens avec les internes et les psychiatres permettraient de mettre en évidence l'évolution des positionnements par rapport à I'héritage d'Henri Collomb et de l'école de Fann au-delà d'une rhétorique de déférence.

3 Sur l'histoire de la psychiatrie coloniale en AOF et la création de l'école de Fann, se référer aux travaux de Collignon (2006), ainsi qu'à la Revue de psychopathologie africaine. Voir aussi : Gueye (1995) et Lachaise (2014).
} 
en charge inclut les indigènes, car ils soulignent que la folie a des expressions locales. L'assistance médicale concerne les indigènes, le groupe des Européens, l'armée et les criminels. Reboul et Régis proposent différents modes d'action afin d'améliorer le système de soins en Algérie, en Tunisie, à Madagascar, en Indochine et en AOF : promulgation de la loi française de 1838 en I'adaptant aux conditions locales, construction d'asiles dans des localités stratégiquement situées au regard des infrastructures de communication et des mobilités des régions concernées, création d'un corps médical spécialisé, arrêt de l'envoi des patients indigènes dans les asiles en France où ils décèdent fréquemment, révision des conditions de rapatriement des civils et des militaires, prévention par des mesures de prophylaxie générale et d'hygiène, et enfin la lutte contre les préjugés dans les cultures locales et l'éducation des populations européennes.

La mise en œuvre de ces recommandations n'est pas réalisée de manière identique en Algérie et en AOF, ces régions faisant I'objet d'investissements spécifiques de la part de l'État. La Première Guerre mondiale puis la période de I'entre-deux-guerres ne permettent pas des avancées significatives en matière d'équipements sanitaires et d'amélioration de la prise en charge des malades en AOF faute de moyens et d'une réelle volonté politique. Durant les années 1920 et 1930, divers projets d'installation d'asiles sont présentés, mais aucun d'entre eux n'aboutit. Le modèle de l'asile ne $s^{\prime} y$ impose pas avec la même vigueur qu'au Maghreb où il est combattu par les milieux médicaux et intellectuels, et ce au-delà de la figure emblématique de Franz Fanon (Keller, 2001; Cooper et Stoler, 1997). Un siècle après la métropole, le 28 juin 1938, I'Assistance psychiatrique indigène (API) est créée. La Seconde Guerre mondiale ralentit le plan prévu et ce n'est qu'en 1956 qu'est créé le premier service de psychiatrie à Dakar.

Entre 1959 et 1978, le médecin français Henri Collomb a la charge de la première chaire de psychiatrie à I'Université. II engage une collaboration entre médecins, tradipraticiens et anthropologues afin de comprendre les représentations et les croyances associées à la folie. Ils développent des pratiques thérapeutiques (le "Penc", l'accompagnant, les villages thérapeutiques) qui mettent en œuvre ses préceptes selon lesquels "on est toujours fou dans sa culture " et que l'hôpital psychiatrique ne doit pas être coupé de la société. Cette conception d'une psychiatrie "culturelle" ou "sociale" reste une revendication des psychiatres sénégalais qu'ils formulent individuellement avec quelques nuances. Après l'indépendance, la loi 75-80 du 9 juillet 1975 sur le traitement des maladies mentales définit le cadre juridique de la psychiatrie au Sénégal. Le seul hôpital psychiatrique du pays est créé àThiaroye dans la banlieue industrielle de Dakar. À Dakar, le Centre hospitalier national universitaire (CHNU) et I'hôpital militaire principal offrent des services en psychiatrie. D'autres unités thérapeutiques beaucoup plus réduites et dépourvues de ressources financières et humaines sont mises en place à Saint-Louis, Ziguinchor (consultation itinérante à Kolda) et Tambacounda. À Thiès et Fatick (consultations itinérantes à Louga et Diourbel), les centres catholiques de santé mentale Dalal Xel sont liés par une convention au Ministère de la Santé et de l'Action Sociale (MAAS). L'offre demeure insuffisante et est très inégalement répartie sur le territoire puisque la région de Dakar concentre $80 \%$ des ressources. 


\section{Dispositif d'enquête}

II n'existe pas de statistiques nationales permettant de mesurer la prévalence des maladies mentales dans la population au Sénégal et encore moins dans des sous-populations spécifiques ${ }^{4}$. Les données de routine (registre de consultations, registre des hospitalisations) des services psychiatriques ne renseignent pas le statut migratoire qui n'est pas inclus dans les caractéristiques sociodémographiques des patients (sexe, âge, situation matrimoniale, profession, localisation résidentielle, ethnie, niveau scolaire). Le lieu de résidence permet dans le cas des malades étrangers d'observer les mobilités thérapeutiques. Par exemple, au centre Émile Badiane à Ziguinchor, la présence de malades venant de Guinée Conakry, de Guinée-Bissau et de Gambie est avérée. Ils représentent environ $7 \%$ des patients qui viennent consulter. Dans les centres psychiatriques de Thiès et de Tambacounda, ce sont des malades en provenance de Mauritanie et du Mali qui se signalent. Cet état de fait révèle l'existence de parcours thérapeutiques transnationaux complexes, en partie expliquée par la faiblesse de l'offre de soins en santé mentale en Afrique de l'Ouest. En ce qui concerne les migrants internationaux sénégalais, c'est le plus fréquemment leur résidence au Sénégal qui est enregistrée, car s'ils sont en vacances ou de retour ponctuel au pays elle est déclarée par l'accompagnant et la temporalité de ce retour est floue.

Le passé migratoire d'un patient peut être appréhendé à travers la lecture de son dossier médical. En effet, le psychiatre lors de l'entretien clinique avec un malade et son accompagnant établit "le tableau médical" en recherchant "les antécédents». Par des questions, il tente de recomposer la biographie (éducation, formation et profession, expérience migratoire, son milieu familial, environnement religieux et culturel) de son patient. Le psychiatre s'adresse toujours et en premier lieu directement au malade; s'il n'est pas en état de répondre, le médecin se tourne alors vers son (ses) accompagnant(s). Lors des consultations auxquelles nous avons assisté ou de discussions sur un coin de bureau avec des praticiens, ils nous ont souvent spontanément proposé d'examiner les dossiers médicaux des patients afin d'expliquer leur démarche médicale et les difficultés du suivi thérapeutique. Indépendamment de questions éthiques évidentes, l'exploitation des dossiers médicaux se révèle difficile pour plusieurs motifs : écritures souvent illisibles, abréviations, vocabulaire médical, etc. Les dossiers médicaux s'ils ne détaillent pas en profondeur l'histoire des patients, démontrent néanmoins que la migration internationale est une donnée récurrente dans les récits familiaux des patients qu'ils soient eux-mêmes migrants, migrants de retour ou parents de migrant. Des fiches de suivi sont établies par les services administratifs pour ceux qui rentrent dans le cadre d'un rapatriement sanitaire organisé entre une structure hospitalière du Nord et le CHNU de Fann à Dakar. Nous n'y avons pas eu accès ce qui aurait permis d'avoir des informations plus systématiques sur ces situations, mais sans pour autant pouvoir accéder à ces patients.

4 Au Sénégal, comme dans la plupart des pays en développement, la santé mentale ne constitue pas une priorité en santé publique malgré la charge morbide qu'elle représente et son impact sur le développement du pays (Charlson et al., 2017; Lund et al., 2011; Patel et al., 2007). 
L'enquête qui articule démographie et anthropologie se déroule depuis 2015 dans différents services psychiatriques sénégalais : à Fann (clinique Moussa Diop, service de consultations de jour et centre pédopsychiatrique Ker Xaleyi), à I'hôpital psychiatrique de Thiaroye (urgences et service de pédopsychiatrie), au centre catholique de santé mentale Dalal Xel àThiès et sa consultation itinérante à Louga, et au centre de santé mentale Émile Badiane de Ziguinchor. Des observations ont été réalisées à l'accueil des unités de soins, aux urgences, dans les salles d'attente, les lieux de vie des patients (chambres, jardins, couloirs, etc.). Dans plusieurs services (Thiaroye, Louga, Thiès, Ziguinchor), nous avons pu assister aux consultations aux côtés des médecins, ce qui permettait de saisir les interactions entre les soignants, le malade et l'accompagnant, mais également entre les membres d'une famille. Parallèlement aux séquences d'observations, des entretiens semi-directifs ont été conduits avec l'accord des chefs de service et de l'administration avec des accompagnants, des personnels soignants (psychiatres, internes, infirmiers, aide-soignants, pharmaciens, psychologues), de personnes malades au moment d'un rendez-vous ou lors d'une hospitalisation et de tradipraticiens (marabouts, guérisseurs, exorcistes). Des psychiatres et des psychologues ont été également interrogés dans le cadre de leurs cliniques et cabinets privés. Certaines personnes sous traitement ont été également rencontrées en dehors des unités médicales dans un lieu choisi à leur convenance (domiciles, cafés-restaurants, espaces religieux).

Les personnes malades doivent avoir obligatoirement "un accompagnant", c'est-à-dire un proche désigné par la famille afin de rester constamment à leurs côtés durant leur hospitalisation. Ce parent doit s'occuper du malade (toilette, habillement, nourriture, tenir propre la chambre, l'assister lors des rendez-vous de suivi ou des examens médicaux). II assiste aux échanges avec le psychiatre et est chargé de faire de lien entre l'institution médicale et la famille. Néanmoins, certaines familles choisissent de rétribuer une personne extérieure à la parenté afin qu'elle remplisse cette fonction en raison des évolutions dans les modes de vie. Les médecins parlent alors "d'accompagnants mercenaires" (KilroyMarac, 2014; Koundoul, 2015) et l'interprètent comme une forme d'abandon des malades mentaux à l'institution.

Les entretiens semi-directifs ont été réalisés soit directement en français, soit en wolof, en diola ou créole avec l'aide d'un soignant et d'un enquêteur-interprète formé en anthropologie de la santé. Ces échanges avaient pour thématiques centrales: les représentations de la folie, l'interprétation des maladies mentales, la nosologie, la stigmatisation des personnes malades, le parcours thérapeutique et le pluralisme thérapeutique, l'adhésion au traitement psychiatrique, les effets de la maladie sur la situation familiale et sociale du patient. Un accueil très positif nous a été réservé dans l'ensemble des institutions sollicitées, ce qui tient à deux raisons principales. Tout d'abord, les médecins se présentent spontanément comme les héritiers de Henri Collomb en se référant à sa collaboration avec des anthropologues et sociologues ce qui légitime encore aujourd'hui la présence de chercheurs en sciences sociales dans les unités psychiatriques. Ensuite, les malades et leurs accompagnants passent de longs moments à attendre dans un contexte hospitalier où ils se sentent perdus, parfois abandonnés et désemparés. L'ennui, I'incompréhension, l'appréhension face à l'avenir et l'isolement social incitent à partager une situation vécue comme une catastrophe ou un drame. Ce besoin de parler est d'autant plus 
fort au sein des unités de soin, que hors ces murs la maladie mentale demeure fortement stigmatisée.

Cette enquête présente deux limites. L'offre privée de santé mentale n'a été que peu investiguée pour l'instant alors que les migrants les plus favorisés et éduqués peuvent y accéder au moment de retours occasionnels ou une fois réinstallés au pays. Les cliniques et les cabinets médicaux offrant des consultations psychiatriques ou psychologiques sont tous localisés à Dakar. Une consultation auprès d'un spécialiste s'élève au minimum à 25000 FCFA (environ 40 euros) alors qu'une consultation dans un service public coûte environ 5000 FCFA (environ 7,50 euros). Cette dernière donne également accès à des analyses et des médicaments moins chers et à certaines facilités de paiement, voire de gratuité dans les centres catholiques. Recourir à une consultation en secteur privé peut s'expliquer par une (supposée) meilleure qualité des soins, mais aussi parce que les patients recherchent une discrétion absolue afin de préserver leur réputation dans un contexte social où, quelle que soit son appartenance sociale, chacun redoutant d'être associé à la folie. La question de la différenciation sociale ne se pose pas uniquement à travers l'alternative d'un recours à l'offre privée ou publique, puisque même à l'intérieur de cette dernière il existe des écarts importants dans les conditions de prise en charge et de soins que peuvent s'offrir les patients et leurs familles. Si le contexte sociétal évolue, les discours relatifs à I'effacement de la maladie et des malades de l'espace public restent récurrents : "Si vous pouviez attacher les malades à un piquet où vous voulez, même dans l'hôpital, et ne plus les laisser sortir ça serait mieux, qu'on ne les voit plus, ne les entende plus" (requête adressée à un médecin psychiatre de Fann lors d'une intervention publique). Rappelons que les premières structures de soins psychiatriques furent installées au Sénégal en remplacement des lazarets, des léproseries et des morgues.

\section{Un parcours migratoire brisé : le retour du migrant "very very VIP»}

\section{La stigmatisation}

La maladie mentale impacte non seulement la personne malade, mais également l'ensemble de son entourage. Elle constitue une déflagration sociale qui ébranle l'organisation familiale, et de même que la réussite du migrant rejaillit sur sa famille, la honte liée à la folie s'abat sur celle-ci. En effet, relativisant les représentations de sociétés africaines bienveillantes à l'égard de leurs "fous", les personnes désignées comme folles par leur entourage tout comme celles conduites dans les services psychiatriques ${ }^{5}$ font l'objet d'une forte stigmatisation. Elles sont exclues de la commensalité et sont I'objet de violences physiques et symboliques (contention, enchaînement, réclusion). Elles perdent

5 L'institution psychiatrique sécrète une image qui modifie progressivement bien au-delà du consensus de l'entourage, les images traditionnelles du fou et de la folie. Elle fait de celle-ci une maladie. Le fou qui n'est plus assumé par le consensus social et culturel est rejeté vers I'hôpital psychiatrique des anciens colonisateurs pour y être soigné, du moins enfermé, gardé, relégué (Storper-Perez, 1976). "L'introduction d'une nouvelle culture, les transformations sociales rapides et profondes bouleversent obligatoirement le statut du malade et l'image de la maladie mentale. Ce bouleversement peut se faire au détriment du malade" (Collomb et Zempleni, 1965). 
statut, autorité et pouvoir, leur parole est discréditée. Parfois, elles sont abandonnées à leur sort dans la rue (Diagne, 2016). Pour les familles, la maladie mentale est fortement associée à la honte et au déshonneur, car le malade par ses comportements transgresse les usages sociaux, les codes de la civilité en étant trop agité et bruyant, ou au contraire en étant plongé dans un mutisme et un abattement prolongé. II trouble les relations au sein de la famille et avec le voisinage. Incapable de mener une activité rémunératrice avec constance, la personne malade constitue est une charge pour ses proches qui doivent l'entretenir et rechercher des soins.

Dans un contexte de fort pluralisme thérapeutique, l'analyse des parcours thérapeutiques montre que le recours à l'offre psychiatrique intervient presque toujours systématiquement en dernier recours lorsque les autres alternatives ont été épuisées. Les malades "ordinaires" sont conduits en état de crise dans les unités psychiatriques, souvent sous contrainte parce que les voisins ou la famille elle-même ont appelé la police ou les pompiers en milieu urbain ou la gendarmerie en zone rurale. Durant des années de quête thérapeutique où le malade est balloté entre divers tradipraticiens (marabouts, guérisseurs, exorcistes, etc.) et ce quelle que soit la religion des familles et des thérapeutes, l'état de la personne malade empire. Le traitement réservé au malade (mental) est dépendant des autres impératifs familiaux, des ressources du moment, du contexte économique et social, du calendrier des fêtes religieuses (Fassin, 1992). Les personnes malades évoquent fréquemment un sentiment d'insécurité et un manque de confiance vis-à-vis de leur famille, affirmant que celle-ci n'a pas respecté leurs choix, leur a imposé d'autres solutions thérapeutiques ou a cessé de financer leurs traitements médicaux. Ces tensions familiales sont exacerbées par certains symptômes qui suscitent incompréhension, l'effroi et la honte. L'expression de ces symptômes (délire, agitation, agressivité, exhibitionnisme) rompt les codes sociaux, un proverbe wolof n'assure-t-il pas : "il dit la vérité, il est fou» ("dof la »). La manière dont les familles ressentent les symptômes de la maladie mentale contribue aux demandes faites aux soignants :

"L'accent est surtout mis sur les signes qui se donnent à voir, générant une honte par rapport aux autres, plus qu'au vécu intime, à l'éprouvé subjectif du patient, fût-il douloureux pour lui. La dissolution des relations est aussi renforcée par un mode de vie perçu

comme dysfonctionnel, déviant par rapport aux normes sociales. [...] Par contre, des signes de grande souffrance psychique du malade comme l'humeur dépressive, l'inhibition motrice, la culpabilité excessive, retiennent peu l'attention de la famille et sont considérés comme "normaux", moins gênants pour l'entourage et plus en cohérence avec les valeurs sociales de la kersa (pudeur, timidité, déférence) et de sutura (discrétion, respect de la vie intime) très prisées du groupe familial. " (Sylla et al., 2009 : 69-71)

\section{"Une société de la honte "}

Les migrants rapatriés sanitaires arrivent à l'aéroport de Dakar où une ambulance les attend et les conduit en général au CNHU de Fann, qui peut ensuite les répartir vers d'autres unités psychiatriques selon la demande de la famille en fonction de leur lieu de résidence et du coût d'hospitalisation. Ceux qui reviennent par leurs propres moyens sont accueillis par leur famille qui là aussi les emmène le plus vite possible à Fann dans un premier temps, car il n'est pas souhaitable que le migrant malade soit vu par le voisinage. Son état 
de faiblesse ou son incapacité ne doit pas paraître publiquement et donner lieu à des rumeurs qui nuiraient à I'honneur (jom) et couvrirait la famille de honte (rus). De jeunes accompagnants ou des visiteurs (frères, cousins, amis des migrants hospitalisés) disent combien il est difficile, presque insupportable, d'être confronté à la déchéance physique et mentale de leur parent migrant. Assis sur le lit en face de son frère aîné allongé sur son lit, un lycéen témoigne:

" Je voudrais être caché pour ne pas le voir, qu'il ne me voit pas, car c'est blessant de voir mon frère, c'est... c'est l'humiliation... quelqu'un de grand comme lui, entreprenant, là... hébété, les yeux fixes, il bave... presque un animal, une bête quoi !... II ne nous reconnaît plus, il a oublié sa propre maman, il ne sait plus mon nom à moi... Les malades

ici ils marchent comme des robots. Ils te regardent fixement. C'est un monde à part."

Cette transformation, en partie liée aux effets secondaires du traitement, est ressentie comme une déchéance d'autant plus douloureuse qu'elle touche un individu qui avait réussi et jouissait de la reconnaissance des siens. Si le projet du départ peut être dissimulé en raison du poids des superstitions afin d'éviter les sorts, le retour, s'il n'est pas glorieux, est maintenu secret. La honte apparaît de manière récurrente dans les entretiens. Tout ce qui est considéré comme un manquement ou un échec vis-à-vis des attentes sociales est une cause de honte "enkystée au plus profond des individus" (G. B., psychiatre, HPThiaroye) que l'on cherche à masquer. Psychologues et psychiatres évoquent de manière unanime une "société de la honte", construite :

"Non sur la culpabilité, mais sur des processus de culpabilisation. [...] Les gens sont éduqués "au bâton", tout un processus d'inhibition est lancé pour ne pas créer, ne pas penser, ne pas questionner, par contre il faut contrôler, il faut se contrôler, contrôler l'autre. " (KT, psychologue, cabinet privé, Dakar)

Les générations qui se succèdent apprennent que "dans la société, il faut être gôre, c'est-à-dire avoir de la noblesse quel que soit son rang social", la noblesse est entendue comme la grandeur de l'âme, la disposition à avoir de la hauteur et à être généreux :

"Mais comment avoir de la noblesse quand tu es dans la pauvreté, la misère, le dénuement? La honte ronge ces personnes parce qu'elles doivent se débrouiller pour vivre, alors elles peuvent voler, se débrouiller, s'effondrer. Ce sont un peu les Jean Valjean des Misérables, mais sans la partie où ça va un peu mieux. Ce sont les misérables je ne peux dire que cela. " (KT, psychiatre, CNHU Fann)

La honte s'ancre dans un très fort conformisme lié à ce paraître social, où il faut éviter toute attitude, tout propos allant à contre-courant :

"Dans la société, il faut se tenir, il faut être apprêté, guindé. L'investissement dans les vêtements, les colifichets, les accessoires, le fait d'être habillé par son père au moment des grandes fêtes n'a rien d'anodin. [...] On est, on reste scotché les uns aux autres pour lutter contre les angoisses d'effondrement. II ne faut pas dépareiller. On reste coincé."

(KT, psychologue, cabinet privé, Dakar)

"On veut montrer qu'on est bien selon un système de valeurs défini. Mais c'est comme si, à la limite, on s'en moquait de ce que l'on fait intrinsèquement. Le plus important, c'est que les autres sachent qu'on est bien. [...] Oui... [dit dans un souffle] 
être soi... Oui, parce que le groupe a quand même quelque chose de sécurisant, je crois : aller dans le même sens, ne pas se différencier, ne pas attirer l'attention sur soi... Parce que ça coûte d'attirer le regard sur soi tous les jours, ça coûte de faire juger tous ces actes tous les jours. Parce que quand on va dans le sens du grand groupe, on fait les choses comme tout le monde le fait, hein. On a cette sécurité de celui qui ne se distingue pas." (AS, psychiatre, HP Thiaroye)

La famille tient conseil afin d'arrêter en urgence le type de recours thérapeutique et les contributions des uns et des autres à son financement. Le père, la mère, puis les frères, l'épouse du migrant interviennent au sein de ce conseil improvisé en fonction de leur degré de proximité avec le migrant et des arguments qu'ils ont à faire valoir (capital social, niveau d'information, ressources financières). Alors que pour les malades non migrants les discussions peuvent s'éterniser entre les tenants d'une option traditionnelle et ceux d'une solution biomédicale la psychiatrie s'impose comme choix pour les migrants internationaux. En effet, en dépit de la peur et de la honte liées à la fréquentation de l'institution psychiatrique, la biomédecine est perçue - au moins pour un temps - comme la plus efficace afin de rétablir la situation, c'està-dire éviter l'arrêt de la rente migratoire et donc par ricochet l'effondrement des projets qui en dépendent.

Des mères en larmes expliquent qu'elles avaient déjà aidé leurs fils à émigrer, et que dans cette situation désespérée, elles vendront leurs bijoux, mobiliseront leurs ressources pour payer les médicaments et l'hospitalisation pourvu que leur enfant puisse "se relever", expression qui s'oppose à "on est là, on est assis" signifiant l'absence d'une activité rémunératrice et de moyens. Dans la société sénégalaise, les mères sont tenues comme responsables de la réussite de leur enfant. Safietou Diack se réfère ainsi à une expression wolof "liggeeyu ndey, anu doom», qu'elle traduit par "le travail de la mère est le déjeuner de l'enfant» (Diack, $2017: 265)$. L'éducation donnée par la mère produit la valeur de l'enfant, en réussissant grâce à cette attention l'enfant renforcera le prestige et la fierté de sa mère. Elle recevra la reconnaissance de sa famille et de sa belle-famille quand son enfant se mariera. L'honneur individuel est fortement intriqué dans l'honneur familial. Un enfant intériorise qu'il doit se faire honneur à lui-même (suturaal) et à sa famille (teral) par la réussite dont la migration est I'une des voies. Le lien entre le fils migrant et sa mère est souvent souligné :

"II n'y a qu'à voir les relations entre les mères et leurs garçons, elles décident de tout, la mère décide à la place de la femme, elle décide de tout. C'est elle qui décide du mariage de son fils, elle s'installe chez lui, et si elle veut le casser, elle casse tout."

(KT, psychologue, Dakar)

Loin des vertus de savoir supporter, d'avoir le courage d'endurer (mun) avec pudeur afin de préserver l'honneur et d'éviter la honte, les émotions et les sentiments sont extériorisés comme si le service psychiatrique constituait un lieu à part, un bouleversement profond non seulement pour le malade, mais également pour tous ceux qui l'accompagnent. Les observations montrent des migrants arrivant encadrés par des oncles, des frères et des cousins tandis que fréquemment les femmes marchent derrière effondrées et éplorées. Le migrant n'est pas accompagné d'une ou deux personnes comme ordinairement pour les autres patients, c'est une délégation familiale qui se présente, qui rapide- 
ment instaure une pression verbale, presque physique par son nombre, sur les médecins. Avant même que tout diagnostic soit posé, les représentants de la famille demandent de "faire taire" leur malade, de le "remettre en état" grâce "aux injections et aux comprimés" qui sont investis du pouvoir quasi instantané de modifier dans le sens souhaité l'état du malade (Ly et al., 2014). La demande est très explicite : les psychiatres doivent rendre au migrant sa bonne santé dans les meilleurs délais, car l'objectif est qu'il puisse être apte à re-migrer au plus vite. Son retour n'était pas "au programme" et n'étant "pas calculé», il ne peut durer, car il affecte l'économie familiale globale en remettant en cause la redistribution liée aux remises migratoires. Le migrant au cours de cette phase est totalement exclu des discussions familiales. II est en quelque sorte dessaisi du projet migratoire, car la maladie ayant rompu le contrat migratoire, celui-ci ne fait plus l'objet de négociations. Si la réussite érige le migrant en figure centrale de la famille, la maladie mentale créée malaise et distanciation. Ce qui est vrai pour tous les malades l'est plus encore pour les migrants, révélant combien leur statut est lié à l'avoir. Hospitalisé au CHNU de Fann et s'interrogeant sur son avenir, un migrant revenu se faire soigner ici (38 ans, employé dans une grande surface en France, son épouse au Sénégal) explique : "Si tu possèdes rien, tu n'es rien... la famille wolof n'est pas généreuse». Un second enquêté nous rappelle que, si autrefois lors des présentations il était de coutume de s'intéresser à la personne afin de connaître son rang social, aujourd'hui on demande à un étranger ce qu'il fait, ce qui constitue une manière détournée d'estimer ses ressources. La réussite économique devient ainsi un critère primordial de détermination sociale.

\section{Un parcours thérapeutique spécifique : "remettre le migrant en état"}

Les comparaisons montrent que le statut de migrant international offre un accès rapide aux services psychiatriques, à la différence des parcours thérapeutiques longs et chaotiques des autres patients. Cependant, l'intensité de I'investissement sur la santé mentale du migrant est soumise à l'évolution de la maladie. Le migrant incarne au Sénégal une des figures majeures de la réussite. Si dans les années 1950-1970, la promotion sociale était symbolisée par le succès dans les études et l'accès à la fonction publique, à partir années 1980, à la suite des plans d'ajustement structurel les migrations internationales deviennent un nouveau modèle de réussite : "le migrant, c'est un very very VIP ici».

\section{Des soignants en posture difficile}

Les psychiatres expliquent qu'ils doivent faire face et résister aux injonctions des familles, car les impératifs thérapeutiques se heurtent immédiatement à la logique de la rente migratoire. La maladie mentale implique un temps d'hospitalisation et d'observation afin de poser un diagnostic et les traitements s'inscrivent dans une temporalité longue. Celle-ci s'accorde mal avec les exigences des multiples investissements réalisés sur le migrant. Certains médecins soulignent également que les familles sont dans un déni de la souffrance psychique du malade, "ils nous demandent d'être efficaces, qu'il retrouve son état normal». Les médecins interrogent brutalement le coût de l'expérience migratoire reléguant I'apparence sociale au second plan. Des psychiatres expliquent que ce 
sont eux qui doivent rappeler, parfois sévèrement, aux parents présents qui leur demandent instamment de rendre performant leur migrant malade, que celui-ci a vécu l'éloignement, l'isolement, la "plus haute des solitudes " ${ }^{6}$, "l'intensité du déficit relationnel " (Collomb et Ayats, 1962: 596), des conditions de vie difficiles et le poids des responsabilités que la famille fait peser sur lui. Le médecin se place en posture de protection et de défense du migrant malade tandis que la famille exprime une posture plus ambiguë. Certains malades vivent douloureusement le fait que certains membres de leur famille cessent d'incarner le monde protecteur et rassurant qu'ils sont censés constituer en se désolidarisant de leurs souffrances. À leurs yeux, cela constitue une infraction grave au contrat moral qui régit leurs obligations mutuelles.

À la fin des années 1970, la sociologue Storper-Perez (1976) évoquait déjà un "univers de contradictions" afin de décrire les difficultés que les individus éprouvent à se situer dans une société en transition. Elle notait également que l'apparition des troubles mentaux parmi les membres d'une famille oblige ceux-ci à actualiser leurs liens. Plus récemment, une psychologue (NK, centre Ker Xaleyi, CHNU Fann) rendait compte de la difficulté des individus à assumer et articuler les rôles parfois antinomiques dont ils sont les porteurs (être "un bon fils", "un bon migrant", "un bon musulman", un époux moderne, etc.), car ceux-ci les placent face à des dilemmes et des contradictions. Ces choix sont difficiles à effectuer, car ils ont des conséquences directes en matière de relations familiales et sociales. Les individus réalisent avec l'aide des psychiatres et des psychologues que l'émancipation est un processus douloureux, qui implique plus que des discours ${ }^{7}$, des décisions, des actes et une forme de mise en cohérence personnelle. Les praticiens pointent de manière récurrente "le manquement de communication", "la circulation très lente de la communication au sein des familles". Pour un psychiatre de l'HP de Thiaroye, leur rôle est "d'aider les patients à être eux-mêmes", alors que le contrôle social pousse " les gens à aller dans le même sens; c'est comme s'il y avait un comportement routinier à adopter avec tout le monde et ça peut aller très loin... ". Les cadets sociaux ont été confrontés durant toute leur vie à des décisions qui leur ont été imposées par les chefs de famille, sans qu'elles soient justifiées ou expliquées alors qu'elles impactent directement leur existence. Les relations familiales sont donc caractérisées par "le poids des non-dits" et la difficulté "à se différencier du malaise" et "du magma familial». Au fil des entretiens, les migrants dénomment rarement leurs parents, ils évoquent les «ils" et "on" qui incarnent l'autorité familiale. De leur côté, les psychiatres, en critiquant implicitement I'obligation de l'accompagnant, expriment la différence très sensible de l'expression du malade lorsqu'il est seul avec son thérapeute et lorsque son discours est contraint par le regard de son accompagnant. Les psychiatres lors des consultations veillent à toujours donner la parole en premier lieu à leur patient et à ce qu'il ne soit pas interrompu par son ou ses accompagnants. Le respect de cet ordre thérapeutique dans la prise de parole reste difficile à respecter par les membres de la famille tant il contrevient aux règles sociales, il faut toute l'autorité du médecin qui menace d'exclusion les accompagnants rétifs pour

6 Pour reprendre le titre de I'ouvrage de I'écrivain-psychiatre Tahar Ben Jelloun (1977) Paris, Édition du Seuil.

7 Lors d'un échange informel, René Collignon qualifiait la société sénégalaise de "société du commentaire, on commente le commentaire». 
qu'il soit appliqué. La production du récit individuel - y compris migratoire et sa confrontation au récit familial sont des enjeux de premier plan dans les relations familiales.

\section{Dilemmes moraux au sein des familles}

Si le statut de migrant international permet une prise en charge psychiatrique, la confrontation entre les temporalités du traitement et celles des exigences familiales évoquées plus haut révèle les dilemmes moraux des acteurs au sein de la famille. Quand les familles expriment très fortement leur volonté que leur migrant recouvre au plus vite sa performativité, elles ne sont pas uniquement animées par un sentiment utilitariste. À travers des paroles, des gestes d'attention et d'affection s'expriment des formes de reconnaissance et de loyauté vis-à-vis de celui qui a permis par son travail le mieux-être de la famille. Même si les médecins soulignent que les migrants et leurs familles sont fréquemment issus du monde urbain et que leurs expériences du monde occidental les ont rapprochés de la psychiatrie, il n'en reste pas moins qu'ils ne partagent pas le même univers discursif que les psychiatres. Ils expriment une interprétation traditionnelle ou musulmane de l'irruption de la maladie : un esprit (un jinn, un seytaane, un rab) a agressé le malade ou celui-ci est victime d'un maraboutage (liggéey). La causalité de la maladie reste extérieure. Selon Sow (1977) dans la conception africaine l'individu malade apparaît à la fois comme victime et comme informateur du danger qui guette le groupe. Les familles cherchent avant tout le soulagement et l'effacement des symptômes en psychiatrie, l'interprétation de la maladie et l'imputation étiologique ne relèvent pas de la biomédecine. Selon les croyances profanes, un fou ne guérit jamais. Les familles citent fréquemment l'expression : "dof du wér day tane" ("le fou ne guérit pas, mais va mieux»), afin de justifier l'interruption d'un traitement ou une mauvaise observance.

Du côté des malades, les discours expriment l'ambivalence liée à l'idéologie du tekki. Ce vocable exprime la volonté de dénouer des nœuds, de rompre la dépendance, sortir de la pauvreté afin de s'assurer une voie vers la réussite et la reconnaissance de son groupe. Cette pensée est très présente dans la culture de la jeunesse urbaine et wolofisée. Lors d'entretiens réalisés à Fann, les jeunes migrants hospitalisés disent dans la même phrase "vouloir se débrouiller seul" et reconnaître que "la famille est toujours là derrière" exprimant la tension entre un fort désir d'émancipation et un besoin de reconnaissance puissant. Moteur d'autant plus puissant qu'il est sous-tendu par un investissement mystique dans le travail pour ceux qui adhèrent au mouridisme. II est douloureux pour les migrants malades de retomber dans une situation de dépendance vis-à-vis de leurs aînés, alors qu'ils avaient conquis une position de soutien de famille, mais dans le même temps ils expriment les obligations que leurs parents ont à leur égard. La pénibilité de cette situation est exacerbée par le fait que le malade se voit dénier dans son individualité et sa souffrance, ses parents parlent et décident en son nom sans systématiquement lui donner des explications en contrepartie. Si la légitimité à demander de l'aide varie avec le degré de réussite de la migration, la distanciation effectuée, la volonté de "sauver la face " (Pian, 2009), elle apparaît comme une contrepartie légitime à des migrants ayant effectué des transferts jusqu'à I'apparition de la maladie. Cette logique sociale apparaît dans l'enquête à travers des expressions qui reviennent au fil 
des discussions : "c'est à leur tour», car parents "le doivent" (sous-entendu prendre en charge le migrant malade).

La maladie mentale et la crise familiale qu'elle génère actualisent les principes à l'œuvre dans l'économie morale en les mettant à l'épreuve. II ne $s^{\prime}$ agit plus de se situer dans une rhétorique de devoirs et d'obligations, mais de se positionner et d'agir très concrètement aux côtés du patient. Plusieurs malades au cours de cette enquête, qu'ils soient migrants ou pas, ont exprimé l'idée qu'au-delà des souffrances, cette épreuve est aussi l'occasion de reconnaître les véritables liens d'affection qui les unissent aux autres ${ }^{8}$. La notion de contrat migratoire évoquée dans la première partie offre une interprétation de la dynamique des liens entre le migrant et l'institution familiale. Goffman (2010: 230-231) évoquant la question du lien social et les exigences des institutions ne rappelle pas seulement que tout contrat comporte une part d'implicite, mais que " passer un contrat, c'est s'affirmer en tant qu'individu doté d'une personnalité et d'une existence précise. Le contrat le plus insignifiant, qui définit minutieusement les droits et les devoirs d'un individu, repose donc parfois sur un nombre considérable de présupposés qui concernent la personnalité de cet individu. [...] La question qui se pose alors est de savoir comment l'individu assume la définition de lui-même impliquée par ces liens". II souligne que les individus disposent de plusieurs possibilités-limites (faillir ouvertement à ses obligations, rompre le lien, rejeter les implications de son engagement sans que son refus se manifeste ouvertement, se conformer exactement), mais qu'en pratique les individus choisissent la voie la moins coûteuse pour eux, en refusant les positions extrêmes tout en exprimant leurs réticences, mais in fine en remplissant leurs obligations majeures.

Dans les situations qui nous retiennent notre attention, les migrants ne sont plus aptes à se conformer aux attentes de leurs parents tandis que réciproquement ces derniers, parce que la maladie a modifié la personnalité du migrant et donc les attendus tacites du contrat, ne se sentent plus aussi fermement obligés vis-à-vis du malade. Du point de vue des parents, la maladie mentale affecte la capacité des migrants malades à avoir un comportement prévisible et continu garantissant la permanence et la consistance du lien social. Investir sur un frère "qu'on ne reconnaît plus et qui ne nous voit plus primordial [sous-entendu qui ni d'identifie ni ne nomme les siens]" devient alors une question source d'atermoiements et de tergiversations, puisque la réciprocité sera imaginée dans une temporalité jugée trop éloignée, dans un horizon obscurci. Les expressions de solidarité ou l'amplitude des gestes d'éloignement entre le malade et les siens donnent une mesure de l'intensité des liens affectifs et de la sacralité qu'ils revêtent. Si le système d'échanges se réduit aux aspects économiques, le migrant devenu malade a tout à perdre.

L'enjeu est pour les psychiatres que non seulement le patient adhère au traitement, mais également sa famille, et qu'ensemble ils construisent "une alliance thérapeutique». En dépit du fait que la psychiatrie soit le recours d'urgence au retour du migrant, on constate que les médecins peinent à interpeler la famille sur ce qu'est une maladie mentale et sur le sens de la guérison. Les parents souhaitent implicitement que la biomédecine ait le même pouvoir qu'ils prêtent

8 Cette idée est évoquée par Egrot (2007) dans le cas des personnes vivant avec le VIH. 
ordinairement à la médecine traditionnelle (guérir d'un coup définitivement), alors que les psychiatres souhaitent amener les familles à s'interroger sur le sens et la réalité de la guérison. Que signifie être guéri? Est-ce un effacement des symptômes, un arrêt de la crise, une mise sous traitement, une stabilisation, un retour à la vie sociale, un retour à la vie active avec un suivi? Questions qui renvoient toutes à la manière dont le futur du malade est envisagé. Les psychiatres conduisent avec le patient et ses accompagnants une "éducation psychologique" impliquant un travail de convergence sémantique. Cette pédagogie constitue un exercice nécessaire, mais périlleux, car les psychiatres doivent éviter l'écueil d'un discours qui pourrait corroborer la croyance selon laquelle un fou ne guérit jamais vraiment et préparer les familles à modifier leur point de vue sur l'avenir du migrant. Les membres de la famille sont en général assez réticents à s'exprimer et à être d'une certaine manière "inclus dans le problème, c'est-à-dire la maladie" (AK, psychiatre, Ziguinchor) par la démarche psychiatrique qui peut être critique vis-à-vis de l'entourage du patient. En effet, celle-ci a des implications peu compatibles avec les attentes de la famille : cette temporalité thérapeutique est trop longue, une observance précise du traitement, une attention aux conditions de vie, alors que le type de guérison souhaité (recouvrer une santé permettant une re-migration) n'est nullement garanti. Le questionnement réflexif sur le coût psychique et émotionnel de la migration heurte "le déni des familles qui ne sont pas prêtes à entendre la souffrance de leur migrant. Elles ne veulent pas connaître le coût de ce qu'elles possèdent" (FB, psychiatre, CNHU Fann). Aussi, si le migrant ne recouvre pas sa santé rapidement se profile un découragement familial induisant l'arrêt du traitement psychiatrique au profit d'un traitement traditionnel, voire d'un arrêt complet de toute quête thérapeutique, celle-ci pouvant ultérieurement reprendre en cas de crise.

\section{Le mouridisme, présence dans la quête migratoire, présence dans la quête thérapeutique}

Parmi les recours succédant parfois à la psychiatrie il faut mentionner, au regard de l'importance du mouridisme ${ }^{9}$ dans la migration internationale sénégalaise, le fait que certains marabouts guérisseurs revendiquent une forme de spécialisation dans le traitement de la folie. Certains marabouts construisent ce qu'ils appellent des "villages thérapeutiques" reprenant abusivement I'appellation de l'expérimentation thérapeutique initiée par Collomb. Des malades suivant un traitement psychiatrique étaient confiés à une communauté villageoise et étaient suivis par un infirmier partageant la même origine ethnique et locale (Collignon, 1983). Le "milieu» rural est considéré par les psychiatres comme plus sain, car plus proche socialement et moins stressant pour les patients que le contexte urbain décrit comme pathogène. Cette captation de l'héritage de Collomb peut être interprétée comme une tentative de légitimation face à l'institution psychiatrique avec qui les marabouts souhaitent créer un partenariat. Elle $s^{\prime}$ inscrit dans une stratégie plus large où les marabouts intègrent des pratiques biomédicales (médicaments, analyses médicales, fichiers des malades avec un diagnostic reprenant parfois la nosologie psychiatrique) leur permettant à la fois de s'inscrire dans le registre religieux et dans le registre médical scientifique.

9 Un grand nombre de travaux ont été consacrés au mouridisme. On citera outre Bava (2003 et 2005) et Diop (1980 et 1981), Copans (1980), Riccio (2003) et Salem (1981). 
Des observations et des entretiens ont été menés en janvier et juillet 2017 dans les concessions de Serigne Dame Dieng dans le village deTobor et d'Abdul Karim Sane dans celui de Mawa dans les environs de Ziguinchor en Casamance. Ces deux marabouts ont acquis une solide réputation parce qu'ils soignent la maladie mentale et accueillent dans leur concession des malades pour un traitement de longue durée, notamment quand les familles s'en déchargent totalement. Alors que le centre de santé mentale Émile Badiane à Ziguinchor - I'unique centre de Casamance - a une capacité d'accueil d'une trentaine de malades pour un psychiatre et un interne en formation, le marabout Serigne Dame Dieng accueille, certes dans des conditions de logement et de vie sommaires, une soixantaine de malades que les familles lui abandonnent ou qu'il recueille alors qu'ils errent dans les rues. Afin d'accréditer le rôle social et thérapeutique de son action et de pouvoir demander des subsides à l'État ou à des ONG, il a créé l'Association pour la réinsertion des enfants déficients mentaux et les enfants en situations difficiles, reconnue par le ministère des Affaires générales et de l'Administration territoriale. En dépit de cet intitulé, il accueille très majoritairement des adultes, surtout de jeunes hommes, dont d'anciens migrants. Ces personnes sont encadrées par d'anciens malades qui se présentent comme étant guéris grâce au traitement mystique (bains, prières, amulettes) prodigué par le marabout ou aux miracles qu'il a accomplis grâce à la baraka. Ils s'accordent sur "la voie» que doit adopter le nouvel arrivant : il doit se conformer à la discipline des lieux, devenir ainsi un "bon malade" s'il veut voir son état s'améliorer :

"Dans la vie rien n'est gratuit : il faut te battre, ne jamais abandonner, même si c'est une illusion qui s'éteint, même si une douleur blesse tes yeux. II faut toujours avoir la voie, la patience, la volonté.. (Bassirou, disciple de S. D. Dieng)

Bien que le traitement ne soit pas présenté comme une conversion au mouridisme, le malade recueilli doit respecter les prescriptions thérapeutiques établies par le marabout (cure mystique, parfois complétée par des prescriptions biomédicales pour les malades ayant consulté dans un service psychiatrique), avoir une activité physique en travaillant aux champs (arachide et maraîchage) et se plier à l'organisation de la concession, quelles que soient ses convictions religieuses. D'après le marabout, relayé par ses disciples, le travail demandé est adapté à l'état du malade et à ses compétences. L'éthique du travail autrefois orientée par l'activité en migration, est désormais appliquée au souci de soi :

"Ici il faut toujours faire quelque chose, ce qui nous intéresse ici c'est la santé, la guérison. II faut faire des exercices physiques, du sport. On vient de finir le maraîchage,

les oignons. On cultive, on vend. Pour garder sa force, le malade doit travailler. II faut obligatoirement qu'il fasse ça. Ce n'est pas bon de rester à rien faire, de dormir sans rien faire. II faut les stimuler. Le corps, l'esprit. On fait des ateliers couture, soudure, menuiserie, le damier, le jeu. La télévision. On fait ça ensemble."

(Bassirou, disciple de S. D. Dieng)

La discipline individuelle s'inscrit dans une organisation collective où chacun a une place et un rôle bien définis. L'activité physique permet au malade de contribuer à l'économie de la concession, ce qui a pour vertu de lui procurer un sentiment d'utilité et de reconnaissance sociale, et donc d'améliorer sa perception de soi et sa santé mentale. Les malades doivent donc travailler à s'améliorer 
physiquement, moralement et psychiquement à tout instant. Cette « mystique du travail» (Bava, 2003) est décrite comme salvatrice. Alors que les migrants qui arrivent ici sont dans une situation d'échec et d'abandon quasi total, que suite à I'exil migratoire succède un exil familial, ces étapes deviennent des épreuves qui trouveront leur sens ici dans la reconquête de soi et sa destinée.

Le marabout, ses disciples et les malades avec qui nous avons échangé reprennent uniformément un discours opposant "la communauté" à l'extérieur. Dans de nombreuses chambres, l'iconographie mouride est présente, et d'anciens migrants déjà imprégnés de la doctrine mouride disent qu'ils se retrouvent "chez eux" (Bava, 2005). Un migrant revenu de Catalogne il y a trois mois et laissé par sa famille qui ne finançait plus son traitement insiste : "Là, nous sommes une grande famille. On se tient. Ici, là, on est des personnes". Que ce soit en présentant la concession comme une "grande famille" ou "un village thérapeutique" ou "un lieu où on ne chasse ni les chiens ni les oiseaux" signifiant que tout le monde peut y trouver sa place, ses membres insistent sur l'importance du lien entre les individus et le sentiment d'être considérés comme des personnes humaines. Cette insistance est à entendre comme une critique à peine voilée des unités psychiatriques et des familles qui les "traitent comme des animaux ne les respectent pas», comme la peur de n'être plus rien. Lovell et al. (2009 : 214) rappellent qu'un «double fil tisse alors la relation sociale de la maladie mentale : une affinité latérale entre ceux qui vivent des expériences $\mathrm{d}^{\prime}$ anomalie [...], une distance sociale entre les usagers et les habitants du monde des "normaux" ".

"Nous, on est dans la solidarité. On se soutient mutuellement. II faut toujours avoir l'amour de ce que tu fais. En Afrique, on a nos croyances. Certaines choses avec le traitement moderne ça ne peut pas le faire, avec le traitement traditionnel ça peut passer. L'hôpital principal [Dakar], I'hôpital régional, I'hôpital de la Paix [Ziguinchor], à Kénia

[centre de santé mentale à Ziguinchor] tu fais des analyses, mais ils [les médecins] n'ont rien vu. Ils ne peuvent pas t'expliquer. Le marabout il fait la voyance, le listiqar. La mère, elle donne le nom du migrant au marabout, le marabout il peut savoir, il connaît. Le migrant il vient pendant les vacances, ou via un proche on envoie des traitements.

D'autres émigrés viennent directement ici. D'autres, les familles disent qu'elles vont donner 30000 CFA le mois, mais elles oublient leur émigré des mois, des années."

(El Hadj, disciple de S. D. Dieng)

"Nous, on est un centre de réinsertion. Notre satisfaction c'est de libérer les malades. On doit être apte à voir comment le malade évolue, comprendre les cas, comment il vit. C'est le marabout qui fait les soins, il sait s'il est apte à retourner dans la société. Nous, on fait le maximum pour lui. Y'en a qui viennent d'Europe. Lui, là il est passé par un centre psychiatrique en Espagne. Sa famille l'a jeté. L'autre, Chérif a fait le Cameroun, la Libye, I'Italie. Abo, la Côte d'Ivoire. El Hadj, I'Allemagne et l'Amérique.

Landing l'a été pris dans un HP en France, après la Suisse. Comment se comporter vis-à-vis de la société? Vis-à-vis de son prochain? Se comporter comme une personne saine. II faut être tout le temps avec eux. Ne pas les laisser penser. Un esprit sain dans un corps sain. Avec des exercices ils retrouvent leur esprit. II faut la patience." (Marabout Serigne Dame Dieng)

La visite de la concession permet de visiter les chambres que les malades célibataires se partagent (hommes et femmes séparés) et celles des couples. 
Le marabout célèbre également des mariages afin de régulariser les relations qui peuvent s'instaurer entre malades et les naissances qui en découlent. Ces pratiques posent des questions éthiques, thérapeutiques et judiciaires, puisque le marabout se substitue aux institutions politiques et médicales du pays. Cependant, comme le soulignent certains psychiatres, aucune autorité au Sénégal ne vient contester ces pratiques discutables, car ces marabouts occupent le vide laissé par les institutions faute de volonté et de politiques efficaces en santé mentale.

Bien que les institutions concernées (la famille, l'institution médicale psychiatrique, l'organisation mouride) cherchent à se distinguer les unes les autres, un regard extérieur ne manquera pas de noter qu'elles partagent des attentes communes vis-à-vis du malade exprimées dans des registres spécifiques qu'il soit migrant ou non : d'une part, celui-ci doit faire son travail en adhérant au traitement que l'institution lui "offre", il doit d'ailleurs lui témoigner sa reconnaissance et sa gratitude en étant un "bon malade", d'autre part ces institutions partagent l'idée que le malade doit se réadapter à la société et manifester le désir de tenir son rôle dans le jeu social. Goffman (2010 : 219-220) souligne qu'ainsi «les psychiatres voient volontiers ce système comme une sorte de serre sociale dans laquelle le malade entrerait dans un état d'infantilisme et d'où [...] il sortirait à l'état d'adulte réadapté à la société ». Le malade est donc poussé à agir, "à constamment penser en fonction de la réussite ou de l'échec de son moi ", à travailler à "son propre redressement" en acceptant la version que l'institution lui donne de sa disgrâce.

\section{Conclusion : glissement de la figure de la réussite à celle du fou}

L'expérience de la mobilité modifie les relations à l'intérieur de la famille en modifiant les rapports de dépendance et d'autorité à travers les processus de redistribution. S'il réussit, le migrant accède à une nouvelle position familiale ou sociale, ou la renforce, bénéficiant de davantage de reconnaissance et de prestige. La confrontation avec la maladie mentale démontre que ce statut de migrant n'est que momentanément bénéfique en lui permettant d'accéder plus rapidement à des soins psychiatriques. Cette différence avec les malades non migrants n'est pas négligeable puisque dans le cas de ces derniers le recours à la psychiatrie est en général repoussé le plus longtemps possible. Le coût du traitement n'est qu'un élément explicatif parmi d'autres, il convient de ne pas mésestimer l'effet des croyances relatives à la guérison, la proximité discursive et sémantique d'autres alternatives thérapeutiques. Chaque famille repoussera autant qu'elle le pourra l'entrée dans une unité psychiatrique qui marquera d'un sceau indélébile le malade et par contrecoup sa famille. Face à la honte et au déshonneur, il faut donc un moteur puissant pour conduire un malade dans une unité psychiatrique : I'amour (filial, conjugal, fraternel, amical) et l'intérêt, ces deux motivations n'étant pas antinomiques. Dans le cas des migrants malades, ces deux motivations peuvent être exacerbées : la rente migratoire représente un enjeu de taille dans une société encore marquée par la pauvreté et les inégalités, les investissements initiaux puis la redistribution cristallisent les liens entre le migrant et sa parenté. Le migrant qui tient sa légitimité et son prestige du succès économique, voit son statut ébranlé par la maladie mentale parce que 
dans sa chute il entraîne ses proches d'une manière plus visible, plus brutale et sans doute plus impensable pour les acteurs qu'un malade lambda. Alors qu'il avait conquis à différents degrés réussite, reconnaissance, autonomie, émancipation, il se voit soudain ramené du fait de la maladie à une situation pire que celle qui précédait son expérience migratoire. II est privé du droit à la parole, toutes les décisions sont prises pour lui, il dépend totalement du bon vouloir des siens. Le héros glisse vers sa figure antinomique, celle du fou. L'économie psychique familiale en est alors durablement affectée, car outre les émotions violentes, contradictoires, ambivalentes, suscitées par la confrontation directe avec la maladie, cette situation reconfigure les relations intra familiales et leurs expressions.

Ce repositionnement affectif et symbolique ressenti comme une "perte d'humanité» par l'ensemble des patients, l'est encore plus par les migrants. II touche à son comble, quand après avoir vécu une phase de lucidité et de mieux-être grâce à la cure psychiatrique, les migrants voient leur traitement interrompu ou pire sont abandonnés par leur famille chez des marabouts. On est alors face à une situation tragique, puisque nombre de jeunes hommes migrants ont émigré en étant portés par les valeurs des Mourides. La nécessaire réussite par le travail, l'investissement constant dans ce projet migratoire et la pression qu'il implique a participé à la rupture de leur équilibre psychique : surmenage, fatigue, stress, dépression (Ebin, 1990-1991). Rentrés au pays pour être soignés ou poursuivre un traitement médical initié en Europe ou en Amérique du Nord, ils échouent finalement chez un représentant de l'idéologie qui peut avoir contribué à leur situation. Alors que le mouridisme les a portés dans leur quête de prestige, il les accompagne désormais dans une quête singulière où se joue leur survie psychique alors que leur famille, la société, l'État, les ONG se désintéressent d'eux. 


\section{Références bibliographiques}

Bava Sophie (2005) "Reprendre la route " : les relais mourides des migrants sénégalais au Niger, in Laurent Fourchard, André Mary et René Otayek, Entreprises religieuses transnationales en Afrique de I'Ouest, Paris, Karthala, pp. 73-88.

Bava Sophie (2003) De la "baraka aux affaires" : ethos économico-religieux et transnationalité chez les migrants sénégalais mourides, Revue Européenne des Migrations Internationales, 19 (2), [en ligne] consulté le 02/10/2016. URL : http:// remi.revues.org/454

Becker Gary (1964) Human Capital: A Theoretical and Empirical Analysis, with Special Reference to Education, University of Chicago Press, 187 p.

Calvès Anne et Marcoux Richard (2007) Présentation : les processus d'individualisation "à I'africaine", Sociologie et sociétés, 39 (2), pp. 5-18.

Charbit Yves (2008) Les migrations internationales et les transferts de ressources vers les populations des pays en développement, Mondes en Développement, $36(2)$, pp. 53-66.

Charbit Yves (2007) Transferts, retours et développement : données, concepts et problématiques, in Véronique Petit Éd., Les migrations internationales de retour dans la perspective des pays du sud, Paris, édition du CEPED, pp. 44-75.

Charbit Yves et Bertrand Catherine (1985) Enfants, familles, migrations dans le Bassin méditerranéen, Paris, Cahier de I'INED n²110, $194 \mathrm{p}$.

Charlson F. J., Dieleman J., Singh L. and Whiteford H. A. (2017) Donor Financing of Global Mental Health, 1995-2015: Assessment of Trends, Channels, and Alignment with Disease Burden, PlosOne, 3.

Collignon René (2006) La psychiatrie coloniale française en Algérie et au Sénégal : esquisse d'une historisation comparative, Revue Tiers-Monde, 187, pp. 527-546.

Collignon René (1983) À propos de psychiatrie communautaire en Afrique noire. Les dispositifs villageois d'assistance. Éléments pour un dossier, Psychopathologie africaine, 19 (3), pp. 287-328.

Collomb Henri et Ayats Henri (1962) Les migrations au Sénégal : étude psychopathologique, Cahiers d'études africaines, 2 (8), pp. 570-597, [en ligne]. URL : https://www.persee.fr/doc/cea_0008-0055_1962_num_2_8_3257

Collomb Henri et Zempleni Andreas (1965) Maladie mentale et acculturation, Médecine d'Afrique noire, 8, pp. 293-296.

Cooper Frederic and Stoler Ann Laura (Eds.) (1997) Tensions of Empire. Colonial Cultures in a Bourgeois World, Berkeley, University of California Press, 484 p.

Copans Jean (1980) Les marabouts de l'arachide, Paris, Le Sycomore, 279 p.

Coutant Isabelle et Wang Simeng (Dirs.) (2018) Santé mentale et souffrance psychique. Un objet pour les sciences sociales, Paris, CNRS éditions, 416 p.

D’Almeida Ludovic, Ba Moussa, Couloubaly Pascal B., Diop Momar-Coumba, Diouf Mamadou, Guisse Youssouph Mb, Mdodj Mohamed, Mbodji Mamadou, Seck Amadou Moctar, Sow Ibrahima et Sylla Omar (1997) La folie au Sénégal, Dakar, Association des chercheurs sénégalais (publié avec le soutien de la CODESRIA), $346 \mathrm{p}$. 
De Haas Hein (2005) International migration, remittances and development: Myths and facts, Third World Quarterly, 26 (8), pp. 1269-1284.

De Haas Hein and Fokkema Tineke (2011) The effects of integration and transnational ties on international return migration intentions, Demographic research, 25 (24), pp. 755-782, [online]. URL: http://www.demographic-research.org/ Volumes/Vol25/24/

Dia Hamidou (2009) Espaces domestiques, espaces villageois, espaces urbains multi-situés. Cinquante ans de migrations à partir de la moyenne vallée du fleuve Sénégal, thèse en sociologie sous la direction de Jean Copans, Université Paris Descartes.

Diagne Papa Mamadou (2016) Soigner les malades mentaux errants dans I'agglomération dakaroise, Anthropologie \& Santé, 13, [en ligne] consulté le 11/05/2018. URL : http://journals.openedition.org/anthropologiesante/2171

Diack Safietou (2017) L'expérience des "jeunes de la rue" (Faqman) à Dakar : entre marginalisation et reconnaissance sociale, thèse en anthropologie sous la direction de Olivier Leservoisier, Université Lyon 2.

Dime Mamadou Ndongo (2007) Remise en cause, reconfiguration ou recomposition? Des solidarités familiales à l'épreuve de la précarité à Dakar, Sociologie et sociétés, 39 (2), pp. 151-171.

Diop Momar Coumba (1981), Fonctions et activités des "dahira" mourides urbains (Sénégal), Cahiers d'Études Africaines, 21 (81/83), pp. 79-91.

Diop Momar Coumba (1980) La confrérie mouride : organisation, politique et mode d'implantation urbaine, thèse sous la direction de Jean Girard, Université Lyon 2, 273 p.

Ebin Victoria (1990-1991) "Laissez venir à moi vos peuples, vos émigrants exténués"... et je leur donnerai une boite de gélatine en poudre : étude d'un syndrome d'allure somatique parmi les émigrés sénégalais à New York, Psychopathologie africaine, 23 (3), pp. 365-385.

Egrot Marc (2007) Renaître d'une mort sociale annoncée : recomposition du lien social des personnes vivant avec le VIH en Afrique de l'Ouest (Burkina Faso, Sénégal), Cultures et Sociétés, 1, pp. 49-56.

Faist Thomas (1997) The Crucial Meso-Level, inTomas Hammer, Grete Brochmann, Kristof Tamas and Thomas Faist Eds., International Migration, Immobility and Development, Oxford, Berg Publishers, pp. 187-217.

Fassin Didier (2009) Les économies morales revisitées, Annales. Histoire, Sciences Sociales, 64 (6), pp. 1237-1266.

Fassin Didier (1992) Pouvoir et maladie en Afrique, Paris, PUF, 368 p.

Faye Papa Lamine (2006) Analyse d'aspects psychopathologiques de la migration chez les émigrés sénégalais. À propos de 14 cas colligés à la clinique psychiatrique Moussa Diop de Fann, thèse de médecine, Dakar, Université Cheick Anta Diop, $130 \mathrm{p}$.

Faye Papa Lamine, Gueye Momar et Thiam Mamadou Habid (2007-2008) Aspects psychopathologiques de l'émigration. À propos de neuf observations à la clinique psychiatrique du CHU de Fann, Psychopathologie africaine, 34 (1), pp. 85-116. 
Goffman Erving (2010 [1961]) Asiles. Études sur la condition sociale des malades mentaux (présentation de Robert Castel), Paris, Les éditions de Minuit, 447 p.

Gueye Momar (1995) The Interface between Culture and Mental IIIness in French Speaking West Africa, in Ihsan Al-Issa Ed., Handbook of Culture and Mental IIIness: An international perspective, Madison, International UP, pp. 93-112.

Gueye Momar (1989) Santé mentale et migration vers la ville, in Gérard Salem et Émile Jeannée Éds., Urbanisation et santé dans leTiers-Monde :Transition épidémiologique, changement social et soins de santé primaires, Paris, ORSTOM, pp. 297-303.

Guilmoto Christophe Z. et Sandron Frédéric (2000) La dynamique interne des réseaux migratoires dans les pays en développement, Population, 55 (1), pp. 105-135, [en ligne]. URL : www.persee.fr/doc/pop_0032-4663_2000_ num_55_1_7099

Hamès Constant (2003) Kuczynski Liliane, Les Marabouts africains à Paris, Archives de sciences sociales des religions, 124 (26), pp. 103-105, [en ligne] consulté le 30 septembre 2016. URL : https://journals.openedition.org/assr/849

Harbison F. Sarah (1981) Family Structure and Family Strategy, in Gordon F. De Jong and Robert W. Gardner, Migration Decision Making, New York, Pergamon Press.

Harris John R. and Todaro Mickael P. (1970) Migration, Unemployment and Development: A Two-Sector Analysis, The American Economic Review, 60, pp. 126-142.

Keller Richard (2001) "Action psychologique »: French psychiatry in colonial North Africa, 1900-1962, PhD dissertation, Rutgers University, The State University of New Jersey, $370 \mathrm{p}$.

Kilroy-Marac Katie (2014) Of Shifting Economies and Making Ends Meet: The Changing Role of the Accompagnant at the Fann Psychiatric Clinic in Dakar, Senegal, Culture Medicine and Psychiatry, 38, pp. 427-447.

Koundoul Adama (2015) La professionnalisation de I'accompagnement des malades en milieu psychiatrique au Sénégal, Le journal des psychologues, 10 (332), pp. 42-51.

Lachaise Joris (2014) Ce qu'il reste de la folie, France, KS visions et BABEL XIII association, HD, couleur, 93 minutes.

Lacomba Joan (2000) Immigrés sénégalais, islam et confréries à Valence (Espagne), Revue Européenne des Migrations Internationales, 16 (3), pp. 85-103, [enligne].URL: https://www.persee.fr/doc/remi_0765-0752_2000_num_16_3_1742

Lee Everett (1966) Theory of migration, Demography, 3, pp. 47-57.

Lessault David, Robin Nelly et Goerg Odile (2015) Sénégal, in Gildas Simon Dir., Dictionnaire des migrations internationales. Approche géohistorique, Paris, Colin, pp. 471-475.

Lovell Anne, Troisoeufs Aurélien et Mora Marion (2009) Du handicap psychique au paradoxe de sa reconnaissance : éléments d'un savoir ordinaire des personnes vivant avec un trouble psychique grave, Revue française des affaires sociales, 1, pp. 209-227. 
Lund C., De Silva M., Plagerson S., Chisholm D., Das J., Knapp M. and Patel V. (2011) Poverty and mental disorders: breaking the cycle in low-income and middle-income countries, The Lancet, 378, pp. 1502-1514.

Ly Mouhamed, Petit Véronique et Pizzolato Giulia (2014) La migration internationale face à la santé mentale au Sénégal : récits, discours, imaginaires, in Cécile Canut et Catherine Mazauric Éds., La migration prise aux mots, Paris, Le cavalier bleu, pp. 221-237.

Marie Alain (2007) Communauté, individualisme, communautarisme : hypothèses anthropologiques sur quelques paradoxes africains, Sociologie et sociétés, 39 (2), pp. 173-198.

Marie Alain, Vuarin Robert, Leimdorfer François, Werner Jean-François, Gerard Étienne et Tiekoura Ouassa (Éds.) (1997) L'Afrique des individus : itinéraires citadins dans l'Afrique contemporaine, Paris, Karthala, $440 \mathrm{p}$.

Massey Douglas S. (1984) Social Structure, Household Strategies, and the Cumulative Causation of Migration, Population Index, 56 (1), pp. 3-26.

Massey Douglas S., Alarcon Rafael, Durand Jorge and González Humberto (1987) Return to Aztlan. The Social Process of International Migration from Western Mexico, University of California Press, $354 \mathrm{p}$.

Ortigues Marie-Claude et Ortigues Edmond (1966) Fdipe Africain, Paris, Plon, $335 \mathrm{p}$.

Patel V., Araya R., Chatterjee S., Cohen A., De Silva M., Hosman C., McGuire H. and Rojas G. (2007) Treatment and prevention of mental disorders in low-incomes and middle-income countries, The Lancet, 370, pp. 991-1005.

Petit Véronique (2013) Counting Populations, Understanding Societies. Towards an Interpretative Demography, Dordrecht Heidelberg Springer, 208 p.

Petit Véronique (Dir.) (2007) Migrations internationales de retour et pays d'origine, Paris, éditions du CEPED, 208 p.

Petit Véronique et Balde Alhassane (2017) Les migrations des Guinéens vers I'Union européenne : marginalité statistique vs. impact social, Outre-Terre, 4 (53), pp. 96-113, [en ligne]. URL : https://www.cairn.info/revue-outre-terre-20174-page-96.htm

Petit Véronique, Qureshi Kaveri, Charbit Yves and Kreager Philip (2019 [à paraître]) Anthropological demography of Health, Oxford, Oxford University Press.

Pian Anaïk (2009) La fabrique des figures migratoires depuis l'expérience des migrants sénégalais, Journal des anthropologues, 118-119, pp. 249-278.

Riccio Bruno (2003) L'urbanisation mouride et les migrations transnationales sénégalaises, in Adriana Piga Dir., Islam et villes en Afrique au sud du Sahara, Paris, Karthala, pp. 359-376.

Salem Gérard (1981) De la brousse sénégalaise au Boul' Mich : le système commercial mouride en France, Cahiers d'Études Africaines, 21 (81/83), pp. 267-288.

Salem Gérard et Lang Thierry (1993) Transition épidémiologique et changement social dans les villes africaines : approche anthropologique de l'hypertension artérielle à Pikine (Sénégal), Sciences sociales et santé, 11 (2), pp. 27-40.

Sayad Abdelmalek (1998) Le retour, élément constitutif de la condition de I'immigré, Migrations et société, 10 (57), pp. 9-47. 
Sjaastad Larry (1962) The Costs and Returns of Human Migration, Journal of Political Economy, 70 (5), pp. 80-93, [online]. URL: https://www.journals. uchicago.edu/doi/10.1086/258726

Skeldon Ronald (2008) International Migration as aTool in Development Policy: A Passing Phase?, Population and Development Review, 34 (1), pp. 1-18.

Sow Ibrahima (1977) Psychiatrie dynamique africaine, Paris, Payot, 280 p.

Stark Oded (1984) Migration Decision Making: A Review Article, Journal of Development Economics, 14, pp. 251-259.

Stark Oded and Lucas Robert E.B. (1988) Migration, Remittances, and the Family, Economic Development and Cultural Change, 36 (3), pp. 465-481.

Storper-Perez Danièle (1976) La folie colonisée, Paris, Maspéro, 156 p.

Sylla Aïda, Ndongo Ndèye Dialé Ndiaye, Fall Lamine et Gueye Momar (20112012) Difficultés de réinsertion familiale de malades mentaux ayant élu domicile à I'hôpital psychiatrique de Thiaroye, Psychopathologie africaine, 36 (3), pp. 317-334.

Sylla Diallo Tabara (2009-2010) Femmes d'émigrées et conjugopathie au Sénégal : une souffrance banalisée, Psychopathologie africaine, 35 (1), pp. 9-24.

Sylla Omar et Mor Mbaye (1990) Psychopathologie et migration : un cas de "wootal ", Psychopathologie africaine, 23 (3), pp. 353-363.

Todaro Mickael (1969) A Model of Labor Migration and Urban Unemployment in Less Developed Countries, The American Economic Review, 59, pp. 138-148.

Zempléni Andrea (1985) La maladie et ses "causes ", L'ethnographie, 2, pp. 13-44. 


\section{Véronique Petit}

\section{Retours contraints de migrants internationaux au Sénégal : dilemmes familiaux face à la maladie mentale}

Cet article expose la situation de migrants sénégalais de retour dans leur pays d'origine en raison d'une maladie mentale apparue au cours de l'expérience migratoire. Alors que le migrant international incarne une figure de la réussite, les effets de la maladie et la temporalité du traitement modifient son statut de héros. Dans le cadre d'une économie morale, la famille est progressivement confrontée au dilemme de rester loyale au migrant en prenant en charge le coût de sa santé ou de renoncer à investir sur un migrant considéré de plus en plus comme un malade sans avenir productif/performatif. Dans un contexte où la maladie mentale est source de honte et de déshonneur, certains migrants malades sont abandonnés par leur famille. Ils trouvent refuge chez des marabouts mourides spécialisés dans le traitement des maladies mentales. Conformément à la doctrine du travail mouride, les activités physiques et psychiques déploient des vertus thérapeutiques et sont salvatrices. Les souffrances de l'exil et les épreuves liées à la maladie prennent alors sens dans le travail thérapeutique engagé.

\section{Forced Returns of International Migrants in Senegal: Family Dilemmas Facing Mental IIIness}

This article describes the situation of Senegalese migrants returning to their country of origin because of a mental illness that appeared during the migratory experience. While the international migrant embodies a figure of success, the effects of illness and the temporality of treatment alter his status as a hero. In the context of a moral economy, the family is gradually faced with the dilemma of remaining loyal to the migrant by bearing the cost of his health or giving up investing in a migrant considered more and more as a patient with no productive future. In a context where mental illness is a source of shame and dishonor, some sick migrants are abandoned by their families. They find refuge with murid marabouts specialized in the treatment of mental illnesses. In accordance with the doctrine of labor mouride, the physical and psychic activities deploy therapeutic virtues and are salvatrices. The sufferings of exile and the hardships linked to the disease make sense in the therapeutic work undertaken.

\section{Retornos forzosos de migrantes internacionales a Senegal: dilemas familiares frente a la enfermedad mental}

Este artículo describe la situación de los migrantes senegaleses que regresan a su país de origen debido a una enfermedad mental aparecida durante la experiencia migratoria. Mientras que el migrante internacional encarna una figura de éxito, los efectos de la enfermedad y la temporalidad del tratamiento cambian su condición de héroe. En el marco de una economía moral, la familia se enfrenta gradualmente al dilema entre permanecer leal al migrante soportando el coste de su tratamiento médico o renunciar a comprometerse con él, considerándole como un enfermo improductivo. En un contexto en el que las enfermedades mentales son fuente de vergüenza y deshonor, algunos migrantes enfermos son abandonados por sus familias. Éstos se refugian en los marabús murides, especializados en el tratamiento de enfermedades mentales. De acuerdo con la doctrina muridista del trabajo, las actividades físicas y psicológicas están dotadas de virtudes terapéuticas y salvan vidas. Los sufrimientos del exilio y las dificultades asociadas con la enfermedad cobran sentido a partir del trabajo terapéutico realizado. 